\title{
AN UNSUPERVISED LEARNING BASED APPROACH FOR UNEXPECTED EVENT DETECTION
}

\author{
Bertrand Luvison, Thierry Chateau \\ LASMEA, Blaise Pascal University, Aubière, France \\ bertrand.luvison@lasmea.univ-bpclermont.fr, thierry.chateau@lasmea.univ-bpclermont.fr \\ Patrick Sayd, Quoc-Cuong Pham \\ CEA, LIST, Embedded Vision Systems Laboratory, Boîte Courrier 94, F-91191 Gif-sur-Yvette, France \\ patrick.sayd@cea.fr,quoc-cuong.pham@cea.fr \\ Jean-Thierry Lapresté \\ LASMEA, Blaise Pascal University, Aubière, France \\ jean-thierry.lapreste@lasmea.univ-bpclermont.fr
}

Keywords: Motion learning, kernel machine, video surveillance.

Abstract: This paper presents a generic unsupervised learning based solution to unexpected event detection from a static
uncalibrated camera. The system can be represented into a probabilistic framework in which the detection is
achieved by a likelihood based decision. We propose an original method to approximate the likelihood function
using a sparse vector machine based model. This model is then used to detect efficiently unexpected events
online. Moreover, features used are based on optical flow orientation within image blocks. The resulting
application is able to learn automatically expected optical flow orientations from training video sequences
and to detect unexpected orientations (corresponding to unexpected event) in a near real-time frame rate.
Experiments show that the algorithm can be used in various applications like crowd or traffic event detection.

\section{INTRODUCTION}

Surveillance is still essentially performed by human supervisors for visually inspecting video streams from CCTV (Closed Circuit Television) networks. However, the number of camera never stops increasing. Indeed, public areas become more and more crowded but also monitored. In this context, real-time assisting monitoring systems which focus the operator on saliency cameras are more than necessary. This type of systems have to deal with complex scenes and a lot of information for extracting scarce subtle events as in crowd or traffic monitoring.

Crowd analysis is a very complex problem due to the variety of events to be detected and the difficulty of modelling them. Examples of events to detect in these crowded scenes may be abnormal trajectories, person falls, panic movement, fighting or dangerous trajectories and unadapted speed in the case of traffic monitoring. Moreover, the solution must be a realtime system since events have to be detected on the fly. In order to reduce the scope of possible events, researchers have generally addressed specific applications. For example, some systems focus on detecting intrusion in a forbidden zone, while others are dedicated to wrong way movement or motionless entities detections.

In this paper, we propose a generic unsupervised learning based system for detecting unexpected motions by vision within a structured area (traffic monitoring for example). The system works on an efficient and compact representation of the model learnt adapted for real-time execution. Moreover, the learning method is fully automatic. It does not need neither any calibration nor any labelled learning database. The next section briefly sums up existing systems dealing with crowd monitoring, Section 3 describes our learning method and its application to the unexpected event detection. Finally, performances of the system will be evaluated in Section 4, both on the quality of the leanrt model and on the result obtained in real situation over a long time testing.

\section{RELATED WORK}

Crowd monitoring for unexpected event detection by vision can be seen as a classification problem, where each image of a video sequence must be classified into one of the two classes: expected and unexpected event. Related works can be divided into two categories: model based approaches and learning 
based approaches.

To analyse crowd, some approaches require preliminary analyses and treatments for extracting more elaborated features, usually found thanks to model based approaches. These features would then be analysed afterward for detecting unexpected events. For example, the ellaborated features could be the people of a crowd or their trajectories. In (Zhao and Nevatia, 2004), a geometric model (ellipse or omega shapes) to detect body and legs or heads and shoulders into crowd images is proposed. Fourier descriptors were also used to characterize human shapes (Dong et al., 2007). The segmented shapes are then tracked and pedestrian trajectories are estimated in order to analyse people behaviour. Other researchers mainly interested in trajectories of entity in a crowd, postulate that the different points tracked from a given person roughly follow the same trajectory at the same speed. These points are gathered either with a bayesian framework as in (Brostow and Cipolla, 2006) and (Li and $\mathrm{Ai}, 2007$ ), or with the RANSAC algorithm (Rabaud and Belongie, 2006). Hybrid methods combine both approaches to segment the crowd, in order to make the system more robust as in (Sidla and Lypetskyy, 2006). In the case of traffic monitoring, $\mathrm{Hu}$ (Hu et al., 2006) (with the additional hypothesis that an entity cannot partially hide another one), gathers features (spatial ones or trajectories) with Kmeans algorithm. Instead of segmenting the crowd in a set of pedestrians, Some approaches propose a global analysis of the motion of the crowd. In (Velastin et al., 2006), a model of expected motion is built from priors on circulation way for detecting wrong way moving person or suspect motionless people. The image motion is then estimated with optical flow techniques, coupled with an additional foreground extraction step. However, model based approaches are very specific to a given environment and a given application. Moreover, such approaches need priors that are not always available.

In learning based approaches, the model of unexpected events is learnt from a training set. This training set can be built from expected object trajectories (Singh et al., 2007; Junejo and Foroosh, 2007; $\mathrm{Hu}$ et al., 2006). Resulting applications detect unexpected trajectories using a classification framework. Trajectories can also infer interactions between individuals (people separating, coming closer, fighting ...) (Blunsden et al., 2007). In (Andrade et al., 2006), the main components (extracted from a SVD decomposition) of crowd motion are learnt using a Hidden Markov Model (HMM). Other learning machines aim at classifying an entry between two classes which correspond well to the wanted system. These two class learning based classifiers like Support Vector Machine (SVM) (Burges, 1998), Relevant Vector Machine (RVM) (Tipping, 2001) and Boosting (Freund and Schapire, 1996) need both positive and negative samples.

In this paper, we focus on the problem of motion analysis, and especially detecting unexpected events from a video sequence provided by a static camera. Moreover, in order to be as generic as possible, we choose a learning based approach. The output of the algorithm is a binary decision associated to the candidate image (expected or unexpected). Nevertheless, this problem is more complex than a common classification process like SVM or RVM since there is no clear definition of what an unexpected event is, and so no database of negative samples is available. Although, one class SVM could have been used, the features learnt do not spread on a high dimensional space. We propose an unsupervised learning based solution to build a model of expected events from a set of videos with an algorithm simpler than one class SVM.

\section{PROPOSED METHOD}

\subsection{Overview}

Giving an offline video stream from a fixed camera, our system is able to learn from a cumulated optical flow orientation computed into regular blocks of the image, a non-parametric likelihood representation. Since the complexity of non-parametric models depends on the size of the training set, we propose an original approach to build a sparse vector machine based model by selecting only relevant samples for approximating the non-parametric likelihood. Finally, a classification rule is deduced from this likelihood to classify the main orientation on each block during online analysis from the same fixed camera (see the outline on fig.1).

This method has the asset to be completely automatic: no camera calibration is needed, no labelling task has to be done on the learning database. Moreover, our sparse representation is particularly adapted for real-time classification during online analysis.

\subsection{Likelihood Based Decision}

Let $I$ denotes a training video sequence. Each image is split into a set of $N$ blocks and optical flow orientations are associated to each block. $\boldsymbol{Z}^{(n)} \doteq$ $\left(z_{1}^{(n)}, z_{2}^{(n)}, \ldots, z_{K}^{(n)}\right)^{T}$ represents a vector composed by the history of all optical flow orientations. 


\section{Learning step (offline):}

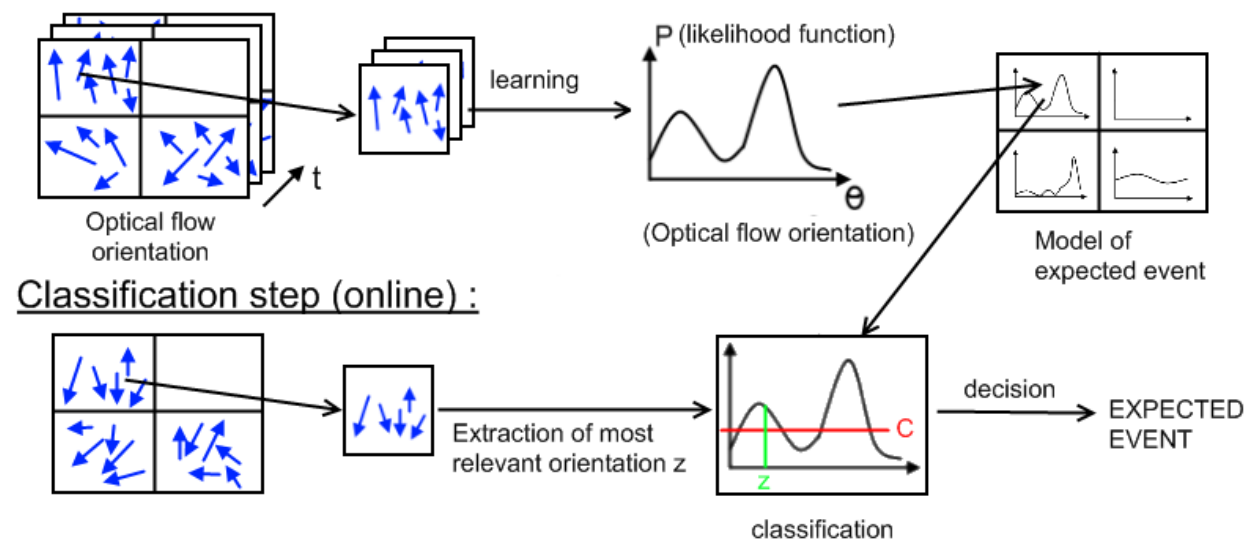

Figure 1: Outline of the system.

We define a Bernoulli random variable $\omega_{k}^{(n)}$ with value in $\left\{\omega_{1} ; \omega_{2}\right\}: \omega_{k}^{(n)}=\omega_{1}$ if the event associated to the block $n$ is an expected event and $\omega_{k}^{(n)}=\omega_{2}$ if the event associated to the block $n$ is an unexpected event. Since nothing is known on the unexpected event likelihood function $P\left(z_{k}^{(n)} \mid \omega_{2}^{(n)}\right)$, we choose to define as unexpected event, observations which cannot be sufficiently reliably assigned to the expected likelihood function $P\left(z_{k}^{(n)} \mid \omega_{1}^{(n)}\right)$. In other words, observations with too low values in the expected likelihood distribution are considered as unexpected.

$$
\widehat{\omega}^{(n)}=\omega_{2}^{(n)} \text { if } P\left(z_{k}^{(n)} \mid \omega_{1}^{(n)}\right)<C
$$

where $\mathrm{C}$ is a detection threshold experimentally determined.

The likelihood $P\left(z_{k}^{(n)} \mid \omega_{1}^{(n)}\right)$ can be represented either by parametric or non-parametric models.

In the first case, GMMs (Gaussian Mixtures Models) are widely used to approximate the likelihood. For example Stauffer and Grimson in (Stauffer and Grimson, 2000) used a fixed number of gaussians GMM for background substraction. EM (Expectation Maximization) algorithms are generally used for GMM parameters estimation but without restrictions on the number of gaussians in recent version (Figueiredo and Jain, 2002). Recently, Han in (Han et al., 2008) proposed a mean-shift based approach to approximate a given distribution with GMMs.

Non parametric models approximate the distribution from samples using kernel based estimation (Parzen windows model (Duda et al., 2001)):

$$
\widehat{P}\left(z_{k}^{(n)} \mid \omega_{1}^{(n)}\right) \approx K^{-1} \sum_{k^{\prime}=1}^{K} \phi_{k^{\prime}}^{(n)}\left(z_{k}^{(n)}\right)
$$

where $\phi_{k^{\prime}}^{(n)}\left(z_{k}^{(n)}\right)$ is a kernel function (not necessarly gaussian). With such a model, no assumption needs to be done over the number of mode of the distribution. Nevertheless, one of the drawbacks of this model is that the estimation of the probability is proportional to the number of samples used. Reduced models can be designed by randomly selecting a subset of $\boldsymbol{Z}^{(n)}$. We propose another solution wherein a sparse model is obtained by approximating eq. (2) by a weighted sum of basis functions. In order to be as generic as possible, we choose the non parametric model instead of GMM aiming at being able to adapt to more atypical distribution. More specific kernel functions may be more appropriated than classical gaussians for these distributions.

\subsection{A Sparse Vector Machine Model for Density Estimation}

Equation 2 can also be expressed with the compact form:

$$
\widehat{P}\left(z_{k} \mid \omega_{1}\right) \approx \boldsymbol{w}^{T}\left(\boldsymbol{\phi}\left(z_{k}\right)\right)
$$

with $\boldsymbol{w}^{T}=(1, \ldots 1)^{T} / K$ is a vector of size $K$ and $\boldsymbol{\phi}\left(z_{k}\right)$ is a vector function defined by $\boldsymbol{\phi}\left(z_{k}\right)=$ $\left(\phi_{1}\left(z_{k}\right), \phi_{2}\left(z_{k}\right), \ldots, \phi_{K}\left(z_{k}\right)\right)$. For the sake of clarity, the bloc index has been left out. This model has the same form as models used for Support Vector Machines (Burges, 1998) or Relevant Vector Machines (Tipping, 2001).

We propose to obtain a sparse model from equation (3) by fixing most of the parameters of $\boldsymbol{w}$ to zero as it is classically done. Let $\Phi$ denote, a matrix of size $K \times K$ associated to the block $n$ and built such as the element of the line $i$ and column $j$ is given by $\Phi_{i, j}=\phi_{i}\left(z_{j}\right)$. $\Phi$ is a square and symmetric ma- 
trix, from which, an estimator of the likelihood associated to the sample $z_{k}$ of the training set is given by $\widehat{P}\left(z_{k} \mid \omega_{1}\right) \approx\left(1_{1, K}\right) \Phi\left(0_{1, k-1}, 1,0_{1, K-k+1}\right)^{T}$ (the sum of the elements of the line or the column $k$ of $\Phi)$. A likelihood vector $\varphi$ related to the training set is built:

$$
\boldsymbol{\varphi}=\Phi\left(1_{K, 1}\right) / K
$$

The sparse model is built in a two step recursive process:

1. The sample of the training set which has the maximum likelihood is selected from $\varphi$ and considered as a relevant vector:

$$
v(m)=\arg \max _{i} \varphi_{m, i}
$$

where $\varphi_{m, i}$ is the $i^{t h}$ element of $\boldsymbol{\varphi}_{m}$ and $\boldsymbol{\varphi}_{m}=\boldsymbol{\varphi}$ for $m=1$.

2. The likelihood estimator is updated:

$$
\boldsymbol{\varphi}_{m+1}=\boldsymbol{\varphi}_{m}-\frac{\boldsymbol{\varphi}_{m, v(m)}}{\Phi_{v(m), v(m)}} \boldsymbol{\phi}\left(z_{v(m)}\right)
$$

Steps one and two are repeated until $m=M$ such as $\varphi_{M, v(M)} / \varphi_{1, v(1)}>t_{v}$. The parameter $t_{v}$ represents the precision of the likelihood approximation. For a coarse approximation $t_{v}$ can be increased. In this case the number of used vectors decreases. Illustration of the effect of this parameter is given in section 4.1. Finally, the estimator is written by:

$$
\widehat{P}\left(z_{k} \mid \omega_{1}\right) \approx \tilde{\boldsymbol{w}}^{T} \tilde{\boldsymbol{\phi}}\left(z_{k}\right)
$$

with

$$
\tilde{\boldsymbol{w}}^{T}=\left(\frac{\varphi_{1, v(1)}}{\Phi_{v(1), v(1)}}, \frac{\varphi_{2, v(2)}}{\Phi_{v(2), v(2)}}, \ldots, \frac{\varphi_{M, v(M)}}{\Phi_{v(M), v(M)}}\right)
$$

and

$$
\tilde{\boldsymbol{\phi}}\left(z_{k}\right)=\left(\phi_{v(1)}\left(z_{k}\right), \phi_{v(2)}\left(z_{k}\right), \ldots, \phi_{v(M)}\left(z_{k}\right)\right)^{T}
$$

This approach enables to give a good approximation of the likelihood with few vectors. Initially, the non parametric model set $\boldsymbol{Z}$ contained $K$ elements whereas the sparse vector machine model $\widetilde{\boldsymbol{Z}}=$ $(v(1), v(2), \ldots, v(M))$ contains only $\mathbf{M}$ elements with $M \ll K$. Let us remember that this reduction in size is compulsory since it makes the real-time classification possible.

\subsection{Online Classification Step}

Online classification based on equation (1) which can be rewritten with our sparse model by :

$$
\widehat{\omega}=\omega_{2} \text { if } \tilde{\boldsymbol{w}}^{T} \tilde{\boldsymbol{\phi}}(z)<C
$$

For the area $n$, a set of optical flow orientation is computed. The most relevant vector $z$ is then estimated using equation (5) by changing $Z$ by the set of optical flow orientations associated to the area $n$.

\section{Experiments}

This section presents the experiments done in order to validate the proposed method. The first part shows the ability of the sparse model to approximate the distribution of optical flow orientation. The second part discusses the performances of the algorithm in two cases of traffic monitoring.

\subsection{Non Parametric Representation}

We make the common choice to use Gaussian data-centred basis functions, $\phi_{k^{\prime}}^{(n)}\left(z_{k}^{(n)}\right)=$ $\frac{1}{\sigma_{l} \sqrt{2 \pi}} \exp \left\{-\frac{d_{\theta}^{2}\left(z_{k}^{(n)}, z_{k^{\prime}}^{(n)}\right)}{2 \sigma_{l}^{2}}\right\}$. Moreover, $d_{\theta}\left(z_{k}^{(n)}, z_{k^{\prime}}^{(n)}\right)$ is a distance between two angles defined by :

$d_{\theta}\left(\theta_{1}, \theta_{2}\right)=\frac{\min \left(\left(\theta_{2}-\theta_{1}\right),\left|\theta_{2}-\left(\theta_{1}+2 \pi\right)\right|\right)}{\pi}$ with $\theta_{1}<\theta_{2}$

The sparse non parametric approximation $\widetilde{\boldsymbol{Z}}^{(n)}$ presented before has been compared to the expanded one $\boldsymbol{Z}^{(n)}$. To make this comparison, the theorical probability representing the non parametric estimator of the likelihood of equation (2) has been represented with the yellow area limited by the black dashed curve on fig. 2. The sparse probability $\widetilde{\boldsymbol{Z}}^{(n)}$ has been drawn for several values of $t_{v}$ with the blue, red and green curves on fig. 2 .

The sparse kernel based model curves on fig.2 show that our reduced representation $\widetilde{\boldsymbol{Z}}^{(n)}$ match the theorical probability. The variance parameter $\sigma_{l}$ should be chosen large enough to have a sufficient decrease in the number of selected vectors with a correct approximation, but also not to big to separate different modes. Experimentally a value of $\sigma_{l}=0.1$ has been chosen. Concerning $t_{v}$ which determines how many selected vectors will be kept or in other words the precision of the likelihood approximation. We can see on the two mode distribution of fig. 2 that for high value of $t_{v}$ (blue and red curves), the bandwidth of the sparse density approximation mode is truncated compared to the non parametric density. Smaller modes can even be forgotten (blue curve). The number of selected vectors, that is to say the number of gaussians needed for the approximation are respectively 1,4 and 8 for the blue, the red and the green curves. For a uniform random distribution, a lot of selected vectors are needed for a correct approximation. Therefore, the parameter $t_{v}$ needs to be small enough to approximate correctly all the orientations possible contrary to the blue curve which underestimates some specific orientations. Here 21, 26 and 39 gaussians have been 

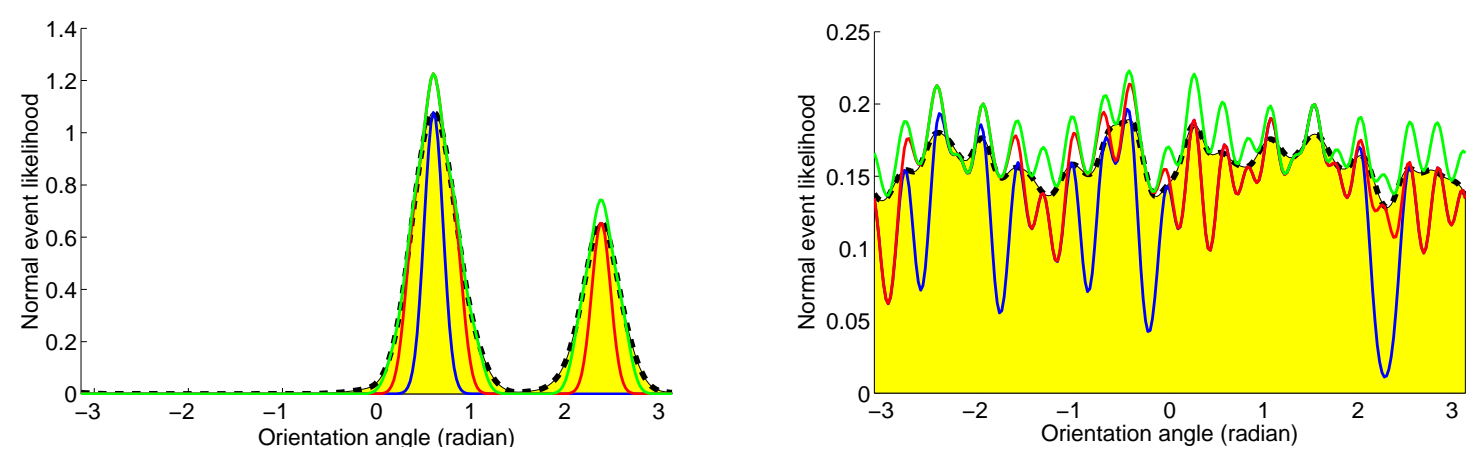

Figure 2: Appoximation of the kernel based non parametric density estimation with a spare kernel base model on two different kind of distribution: The yellow area limited by the black dashed curve shows the kernel based non parametric density estimation. The blue curve $\left(t_{v}=0.7\right)$, the red one $\left(t_{v}=0.4\right)$ and the green one $\left(t_{v}=0.08\right)$ show the sparse kernel based density estimation.

needed for the approximation for the blue, the red and the green curves respectively.

\subsection{Classification}

The performance of the system has been evaluated on two different scenes: a traffic cross-road with clear circulation way and a toll barrier. Sequences for both scenes were captured on real webcams in very various weathers going from sunny to cloudy through rainy condition, but also under very different light conditions such as projected shadows, specular lights, reflects on the ground, etc (e.g. fig.3). Each video of the dataset is a quite low resolution, 320x240 size at about 12 fps with sometimes very poor objective lens. The videos taken every 20 minutes, last about $30 \mathrm{sec}-$ onds each. The learning has been done over one hundred videos. The block are $10 \times 10$ size, that is to say a total of 768 blocks for each image.

For the movement evaluation, different optical flow techniques have been tested, from Lucas \& Kanade and its variants to "Block matching method" (Barron et al., 1992) through Proesmans method (Proesmans et al., 1994) and others. From all the different techniques, the Black \& Anandan method (Black and Anandan, 1996) has been kept for its robustness and the cleanliness of its result compared to others methods, but also for its relative fast computation. This method is based on a piecewise affine motion assumption which is generally satisfied for our type of scene.

Moreover, the use of this techinque makes the system work whichever lighting condition since optical flow techniques are usually invariant to illumation changes.

The classification performance of the system has been evaluated in real conditions. The analysis has
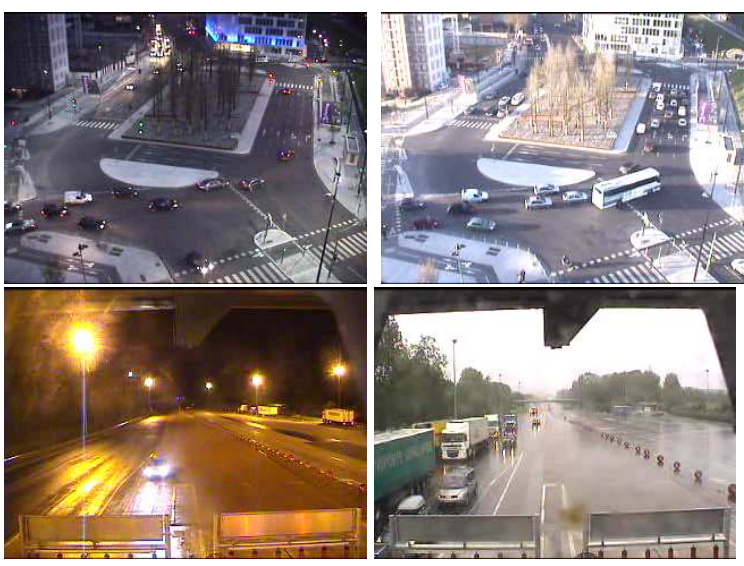

Figure 3: Example of weather conditions in the video database used for learning. Top row images are taken from the traffic cross-road sequence and the bottom one from the toll.

been done over 162 videos for the traffic cross-road and 256 videos for the toll. The anomalies researched were of different types from pedestrians not walking on zebra crossing to scooters leaving the pavement through wrong way mouvement or cars pulling out just before the toll. The classification was done on a P4 3.4GHz. The optical flow is computed in $0.11 \mathrm{~s}$ per frame. The computation time for analysing one block depends on displacement vector density in the block. Non computed for motionless blocks, it takes about $5 \mathrm{~ms}$, that is an average of $0.2 \mathrm{~s}$ per frame. For the sequence studied so far, these computation performances are close to real-time even with a non optimized code. Moreover, the real-time classification lends itself very well to parallelization on graphic cards or multithread architectures in order to increase the framerate. 
An unexpected event is considered to be detected if the system has pointed out at least one block on the guilty object during the unexpected event (e.g. fig.4(d)). False alarms have been counted on the same way, when at least one block appears on an area without true anomaly still during the manoeuvre length (e.g. fig.6). This manual count has been done from the warnings per image provided by the classifier. Even though the system gives a frame warning instead of an event warning (no temporal consistency on events is performed), we choose to present an event count as it is a measure of the performance of the system very close to what it is expected to do. Indeed, the system should alert a security officer when something goes wrong, no matter where the problem occurs which is usually obvious for a human. The system parameters have been chosen in order to have the maximum of good detections with the minimum false alarms.

The parameter $\mathrm{C}$ is deciding to guarantee a low rate of false alarm, the higher it is, the smaller a deviation from a learning orientation will be considered as abnormal. As a consequence, to avoid false alarm due to noise, orientation compution imprecision, etc, parameter $\mathrm{C}$ should be chosen quite low even if correct detections are missed.

With the parameter $C$ equals to $0.3,17$ warnings have been raised over toll sequences total length of 7680 seconds, that is to say an average of one warn every 451 seconds. On these 17 warnings, 16 were real unexpected events whereas the last warning was a false alarm. Traffic cross road sequences is far more harder situation to monitor since the circulation ways are more intricate, the vehicle are smaller, etc. However, the system still warns 7 times over the 5160 s of the total length, that is to say on average of one warn every 737 seconds. Over these 7 detections only 3 were real unexpected events. More events could have been detected for both sequences but with a tremendous increase in the false alarms as shown on table 1. Figures 4(c)-4(f) illustrate the events to detect, the main direction for each guilty block but also the detection conditions for each videos (especially lighting condition). Nevertheless, figures 4(a) and 4(b) show missing detections surrounded with a green square.

\begin{tabular}{|c|c|c||c|c|}
\hline \multirow{2}{*}{$C$} & \multicolumn{2}{|c||}{ Toll sequences } & \multicolumn{2}{c|}{ Cross-road sequences } \\
\cline { 2 - 5 } & CD & FA & CD & FA \\
\hline 0.3 & 16 & 1 & 3 & 4 \\
\hline 0.43 & 28 & 10 & 4 & 38 \\
\hline 0.58 & 38 & 113 & 13 & 164 \\
\hline
\end{tabular}

Table 1: Detection results for different $C$ values. CD stands for Correct Detection and FA stands for False Alarm.
Due to a large number of wrong positive detections that punctually appear, blurrings have been applied to the classifier output. Assuming that an anomaly remains for several frames and spreads from one block to an adjacent one, a temporal blurring has been applied by finding the best relevant orientation among the orientations of the last $k$ frames. Experimentally, using $k=2$ deletes some detection noises due to error in the displacement field computation. Furthermore, a first order temporal filter with a spatial blurring which balances the response of the neighbour blocks has been applied.

Most of the remaining wrong positive detection (wrong unexpected event detected) are mainly due to three reasons: The first one is a possible motion that has not been learnt (this motion has not been seen enough into the training database). An illustration of this, is shown on fig. 5 where the learning orientation has been represented in green. We can see that the learning on this block forgets the relevant orientation towards the left leading to warnings when a car comes from the right. The second reason occurs when the low level (optical flow) provides noisy features. Here, the aperture problem during optical flow computation is the main cause of noisy features. This problem is intrinsic to the way optical flow is computed. This problem often appears on shadows or beams of headlights returning completely wrong displacement direction (e.g. fig.6). To attenuate these unwanted effects, a correlation of the optical flow with an Harris score (Harris and Stephens, 1988) has been done in order to compute the optical flow only on corner features. The third reason is the projection of shadows of objects on the floor. In those case, the shadow generate artefacts, moving in the same direction as the real object, but that could spread over areas with completely different learnt directions (e.g. fig.7). This last problem is hard to deal with.

\section{Perspectives and Conclusion}

We have proposed a generic system for unexpected event detection by vision. The latter learns the orientations of motion on a scene and checks those orientations afterward during live real-time analysis. We have proposed a sparse representation of the distribution of local optical flow orientation by a linear kernel based model. The resulting algorithm has been tested on real sequences and we have shown that the method is able to classify unexpected events like wrong way movement, car pulling out in toll line-up or turning back, etc. Even though the test has been done over traffic sequences, this algorithm can also 


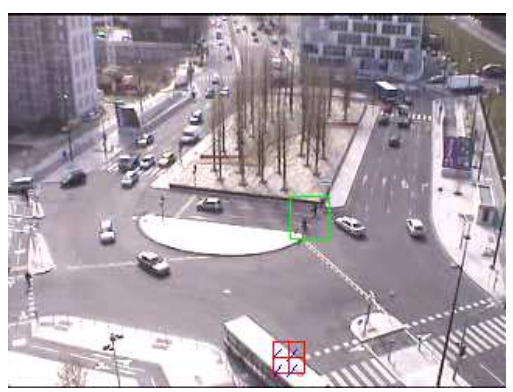

(a)

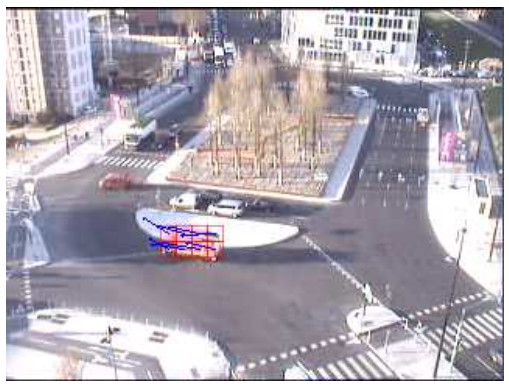

(d)

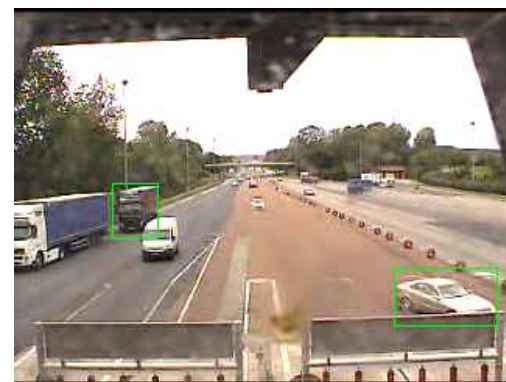

(b)

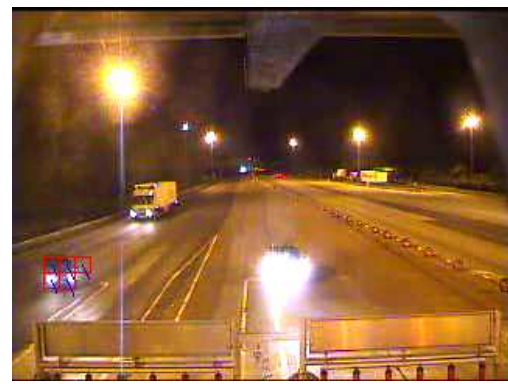

(e)

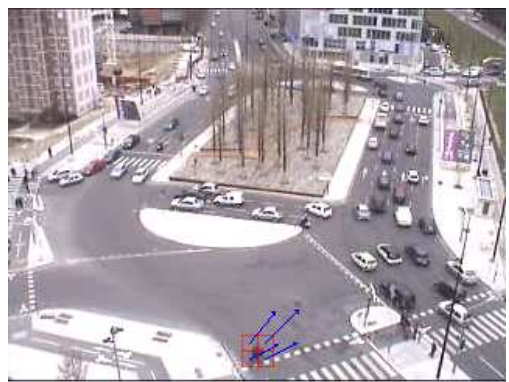

(c)

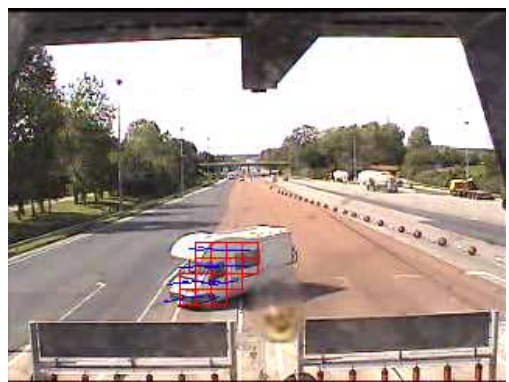

(f)

Figure 4: Different unexpected event samples. The red square represents the unexpected block detected according to the most relevant vector of this block at time $t$ represented with the blue arrow. The green squares point out missed unexpected events. (a) Unexpected pedestrians crossing out of zebras and false alarm example on the bus at the bottom, (b) Double missing detection on a car on the right and on a truck on the left that are both suddenly pulling out, (c) Scooter leaving the pavement following an unsual trajectory, (d) Firefighter truck going wrong way to avoid held up lanes, (e) Car changing direction at the last minute on the left of the camera, (f) Motorhome going across several lanes to reach heavyweight lane

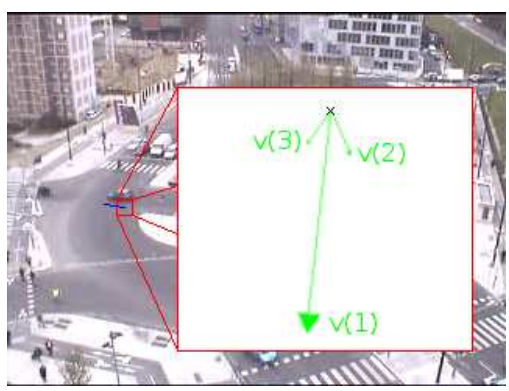

Figure 5: Example of false alarm due to wrong learning orientation. The three vectors kept in sparse model have been represented in green. All of them go downward.

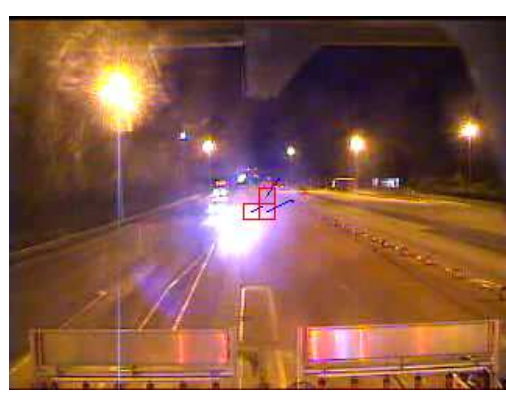

Figure 6: Example of false alarm due to the aperture problem. The optical flow vector are normal to the light beam edge giving completely wrong results.

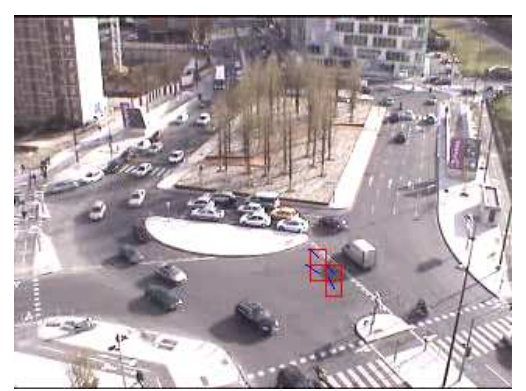

Figure 7: Example of false alarm due to projected shadow. The shadow expands on areas where no movement from down right corner to up left corner are never seen be useful for monitoring pedestrians in indoor corridors as shown on fig.8, where two people are detected going wrong way since the pedestrians normally walk counter clock-wise, that is to say upward on the right side and downward on the left side.

Future work will aim at reducing the wrong detections, often due to noisy features provided by the optical flow algorithm. Several solutions are under consideration : to replace the optical flow by other approaches like detection/matching, to add more efficient temporal blurring in order to have event warning instead of image warning, to make a discussion over the best type of kernel to use and finally to compute an adaptative detection threshold $\mathrm{C}$ in relation to the learnt distribution. Moreover, the system only detects anomalies linked to motion. Expansion to motionless or micro movement detection is also expected for future in order to enlarge the types of anomalies to de- 
tect (person on the ground, suspect parking ...).

This method has been designed in order to be as generic as possible with no supervising, as a consequence it may also be used in completely different fields than video surveillance. We can imagine to use this approach for detecting perturbations on satellite imaging of solar streams for example.

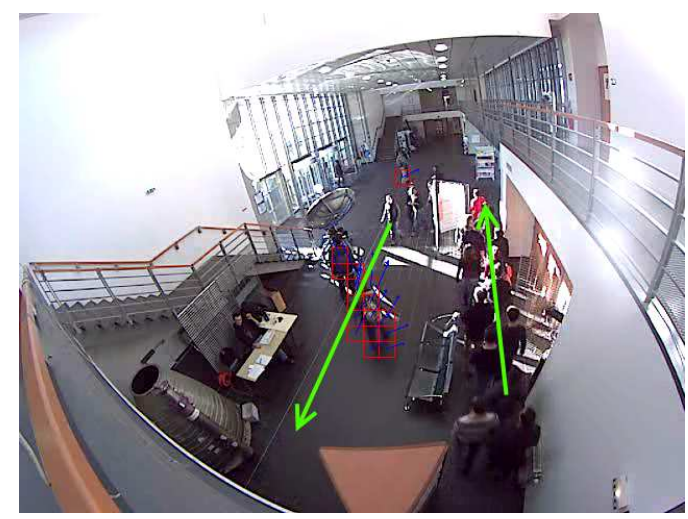

Figure 8: Crowd monitoring example. The expected circulation way has been represented with the green arrows.

\section{REFERENCES}

Andrade, E. L., Blunsden, S., and Fisher, R. B. (2006). Hidden markov models for optical flow analysis in crowds. In International Conference on Pattern Recognition (ICPR), volume 1, pages 460-463.

Barron, J., Fleet, D., Beauchemin, S., and Burkitt, T. (1992). Performance of optical flow techniques. Computer Vision and Pattern Recognition (CVPR), 92:236-242.

Black, M. J. and Anandan, P. (1996). The robust estimation of multiple motions: parametric and piecewisesmooth flow fields. Computer Vision and Image Understanding (CVIU), 63(1):75-104.

Blunsden, S., Andrade, E., and Fisher, R. (2007). Non parametric classification of human interaction. In Pattern Recognition and Image Analysis (PRIA).

Brostow, G. j. and Cipolla, R. (2006). Unsupervised bayesian detection of independant motion in crowds. In Computer Vision and Pattern Recognition (CVPR).

Burges, C. J. C. (1998). A tutorial on support vector machines for pattern recognition. Data Mining and Knowledge Discovery, 2:121-167.

Dong, L., Parameswaran, V., Ramesh, V., and Zoghlami, I. (2007). Fast crowd segmentation using shape indexing. In International Conference on Computer Vision $(I C C V)$.

Duda, R., Hart, P., and Stork, D. (2001). Pattern Classification. John Wiley \& Sons Inc.
Figueiredo, M. and Jain, A. (2002). Unsupervised learning of finite mixture models. Pattern Analysis and Machine Intelligence, 24(3):381-396.

Freund, Y. and Schapire, R. E. (1996). Experiments with a new boosting algorithm. In Proceedings of the 30th International Conference on Machine Learning (ICML), pages 148-156.

Han, B., Comaniciu, D., Zhu, Y., and Davis, L. S. (2008). Sequential kernel density approximation and its application to real-time visual tracking. Pattern Analysis and Machine Intelligence, 30:1186-1197.

Harris, C. and Stephens, M. (1988). A combined corner and edge detector. In Alvey Vision Conference, pages 147-151.

Hu, W., Xiao, X., Fu, Z., Xie, D., Tan, T., and Maybank, S. (2006). A system for learning statistical motion patterns. IEEE Pattern Analysis And Machine Intelligence, 28:1450-1464.

Junejo, I. N. and Foroosh, H. (2007). Trajectory rectification and path modeling for video surveillance. In International Conference on Computer Vision (ICCV).

Li, Y. and Ai, H. (2007). Fast detection of independent motion in crowds guided by supervised learning. In International Conference on Image Processing (ICIP).

Proesmans, M., Gool, L. V., Pauwels, E., and Oosterlinck, A. (1994). Determination of optical flow and its discontinuities using non-linear diffusion. In European Conference on Computer Vision (ECCV).

Rabaud, V. and Belongie, S. (2006). Counting crowded moving objects. In Computer Vision and Pattern Recognition (CVPR).

Sidla, O. and Lypetskyy, Y. (2006). Pedestrian detection and tracking for counting applications in crowded situations. In Advanced Video and Signal Based Surveillance (AVSS).

Singh, S., Machiraju, R., and Parent, R. (2007). Visual analysis of trajectory clusters for video surveillance.

Stauffer, C. and Grimson, W. E. L. (2000). Learning patterns of activity using real-time tracking. IEEE Pattern Analysis And Machine Intelligence, 22:747-757.

Tipping, M. E. (2001). Sparse bayesian learning and the relevance vector machine. Journal of Machine Learning Reasearch 1.

Velastin, S. A., Boghossian, B. A., and Vicencio-Silva, M. A. (2006). A motion-based image processing system for detecting potentielly dangerous situations in underground railway stations. Transportation Research Part C: Emerging Technologies, 14.

Zhao, T. and Nevatia, R. (2004). Tracking multiple humans in complex situations. IEEE Pattern Analysis And Machine Intelligence, 26:1208-1221. 